УДК 316

$10.17213 / 2075-2067-2020-3-118-124$

\title{
СИСТЕМА ДИСТАНЦИОННОГО УПРАВЛЕНИЯ ПЕРСОНАЛОМ: УСЛОВИЯ И СПОСОБЫ ЭФФЕКТИВНОЙ РЕАЛИЗАЦИИ
}

\author{
(C) 2020 г. А. Н. Руденко
}

\section{Ростовский государственный экономический университет (РИНХ), 2. Ростов-на-Дону, Россия}

Целью исследования является раскрытие основных социально-экономических особенностей системы управления персоналом, реализуемой дистанционным способом, разработка базовых рекомендаций для руководителей и подчиненных, способствующих наиболее оперативно справиться с переходом в новый режим взаимодействия, не испытав при этом значимых потерь.

Методологическую базу исследования представляют опыт современных менеджеров, сумевших эффективно реализовать и обозначить непосредственно процесс перевода организации в цифрровое пространство, осветить положительные стороны и подводные камни структурных элементов процесса.

Результаты исследования. Система управления персоналом в условиях дистанционной формы реализации ее основных функиий имеет множество специифических особенностей. Современные организации практикуют дифференщированный подход в управлении «удаленным персоналом», реализуя огромный спектр разнонаправленной деятельности, которому препятствует в значительной степени техническая проблема.

Перспективу исследования составляет дальнейшее соииологическое исследование приобретаемого опыта компаний в поиске новых вариантов выбора эффективных решений.

Ключевые слова: персонал; система управления персоналом; дистанционное управление персоналом; управление «удаленным персоналом».

\section{REMOTE PERSONNEL MANAGEMENT SYSTEM: CONDITIONS AND METHODS FOR EFFECTIVE IMPLEMENTATION}

\section{(C) 2020 A. N. Rudenko}

\section{Rostov State University of Economics (RSUE), Rostov-on-Don, Russia}

The purpose of the study is to disclose the main socio-economic features of the personnel management system, implemented by remote means, to develop basic recommendations for managers and subordinates that help to cope most quickly with the transition to a new interaction mode without experiencing significant losses.

The methodological base of the study is the experience of modern managers who have been able to effectively implement and directly designate the process of transferring an organization to digital space, to highlight the positive aspects and pitfalls of the structural elements of the process.

The results of the study. The personnel management system in the conditions of the remote form of the implementation of its basic functions has many specific features. Modern organizations 
practice a differentiated approach in the management of «remote personnel», realizing a huge range of multidirectional activities, which is largely hampered by a technical problem.

The prospect of the study is further sociological research of the acquired experience of companies in the search for new options for choosing effective solutions.

Key words: personnel: personnel management system; remote personnel management; management of «remote personnel».

Введение. Система управления персоналом, бесспорно, играет ключевую роль в деятельности любой организации. Наиболее эффективным, а следовательно, и результативным способом внутриорганизационного взаимодействия остается личный контакт [1, 2]. К сожалению, иногда возникают ситуации, в условиях которых реализовать данный способ не представляется возможным. Тогда организациям необходимо использовать альтернативные дистанционные варианты для осуществления своей деятельности.

Основные условия реализации дистанционного управления персоналом. Для того чтобы реализовать обыденную трудовую деятельность в условиях дистанционного взаимодействия и контроля и вдобавок не растерять ее количественные и качественные составляющие, необходимо выполнить определенные условия.

1. В первую очередь определить правильного субъекта, способного оказывать техническую и информационную поддержку сотрудникам организации, и установить с ним наиболее тесное взаимодействие.

Иногда не имеет никакого значения, разбирается ли руководитель в тонкостях, порядке и технологии тех или иных процессов. Хороший руководитель обладает одной крайне важной личностной характеристикой - умением управлять людьми, причем, если данная черта у руководителя имеет достаточно высокий уровень сформированности, зачастую теряет свою значимость количественнокачественный состав сотрудников, находящихся в его непосредственном подчинении, поскольку знающий, что необходимо делать с субъектами, управленец сможет настроить рабочий процесс в выгодном для него темпе.

2. Далее в зависимости от конфигурации организационной структуры компании не- обходимо создать объективное количество тематических и функциональных чатов для мгновенного распространения и обмена необходимой информацией.

Формирование подобных информационных групп возможно в таких приложениях, как WhattsApp или Telegram. Определение состава участников может варьироваться в зависимости от различных признаков: уровня власти, структуры функциональных обязанностей, наименования должностей и т.д.

3. Оптимально определять и дифференцировать цели необходимо по средствам командных обсуждений. Опыт рядовых сотрудников заставляет их чаще придумывать более простые способы для решения типичных проблем.

4. Исключить наличие двух линейных руководителей одного уровня при распределении пересекающихся дистанционных функций. К сожалению, при наличии такой проблемы весь трудовой процесс может регулярно тормозиться и застревать. Два руководителя, даже согласованно принимающих одинаковые решения, зачастую имеют собственное видение реализации того или иного действия: его структуры, технологии, пошаговых инструкций и даже речевых формул. Соблюсти указания обоих сторон одновременно становится зачастую невыполнимой задачей.

5. В рабочей группе каждого уровня необходимо обозначить звено, ответственное за взаимодействие с руководством, и при наличии возможности отразить данное решение технически в настройках дистанционных групп. Для чего это необходимо? На практике при наличии огромного состава участников, взаимодействующих по поводу определенного круга вопросов с конкретным структурным звеном, возникает огромное количество сомнительно необходимой для изучения инфор- 
мации. Весьма ограниченную возможность приобретает способность и желание для ее полного изучения. Важные вопросы и ответы рассеиваются и теряются среди многочисленных сообщений, поэтому информация не доходит до адресатов, постоянно возникают одинаковые вопросы, и соответственно, субъектам-профессионалам, обладающим должным уровнем компетенции, приходится тратить огромное количество времени на регулярное решение и пояснение одних и тех же проблем, что значительно увеличивает трудозатраты, длительность рабочего дня, количественный и качественный результат их деятельности.

6. Любой руководитель, желающий управлять своими сотрудниками дистанционно, обязан научиться проводить онлайн конференции, используя двухканальную видеосвязь, сразу с группой подчиненных. Все встречи, которые руководитель проводит на рабочем месте, могут также реализовываться в сетевой среде.

Некоторая деформация коммуникации также эффективна. Стиль делового общения в интернете имеет тенденцию меняться от более формального к менее формальному, что приводит к уменьшению психологической дистанции и как следствие - снижению психологического напряжения.

7. В системе дистанционного управления крайне важно регулярно давать задачи и получать ежедневные отчеты о проделанной работе, иначе отсутствие видимого контроля приведет к снижению темпа трудовой деятельности, работе время от времени и даже прекращению выполнения функциональных обязанностей.

Практика показывает, что при увеличении числа субъектов, имеющих возможность изучить авторский материал, уровень и качество этого материала резко повышаются, поэтому наиболее эффективный способ повышения качества формируемой документации - это создание условий для ее внутриорганизационной и по возможности внеорганизационной прозрачности.

8. Управление эффективностью - это система управления персоналом, основанная на оценке достижения поставленных целей и оценке уровня развития компетенций [3].

Основная проблема современной системы оценки эффективности большинства видов деятельности - это фиксация на количественном показателе. Данная проблема в условиях дистанционного режима совсем не теряет своей значимости, а приобретает новый формат. Основные показатели переводятся в красивые графики, сравниваются ежемесячно, ежеквартально с предыдущими годами и т.д. К сожалению, далеко не для каждого вида деятельности количественный показатель является базовым. Тогда бы для системы управления персоналом бесконечно увеличивающаяся численность сотрудников была бы единственной необходимой к достижению целью.

Эффективные способы реализации дистанционного управления персоналом. Рассмотрим варианты системы управления персоналом в условиях дистанционной формы реализации ее основных функций, примеры и подходы в управлении «удаленным персоналом».

Выбор способа для дистанционного управления зависит в первую очередь от личности руководителя, его компетенции, потому что первый вариант управления - это жесткая связь, а второй вариант - более свободный формат. Более жесткая связь необходима на этапе, когда люди только учатся работать удаленно, тогда нужна жесткая регламентация процессов. А второй вариант подходит, когда сотрудники отдела кадров уже знают все правила игры, пользуются доверием руководителя, тогда это не вызывает лишних вопросов. Это еще зависит от способности управленца делегировать и контролировать, а также от доверия, ведь у некоторых людей есть убеждение, что все нужно постоянно проверять, чтобы люди лучше справлялись с работой, не обманывали, а менеджеры такого типа, естественно, тяготеют к жесткому контролю [5].

Руководитель, жестко контролирующий своих подчиненных, наблюдающий за их поведением, может в один прекрасный момент понять, что эффективность их деятельности на высоком уровне вне зависимости от степени его участия. Этот фактор будет являться первым звоночком для возможности изменения подхода к дистанционному управлению.

Невидимые проблемы дистанционного управления отделом кадров заключаются в том, 
что связь может быть потеряна, потому что когда руководитель на самом деле физически не на месте, он может лишиться чувства владения ситуацией. Современные интернет-технологии наряду с программным обеспечением, позволяющие регулярно взаимодействовать с использованием видеосвязи, приносят огромную пользу, как рядовому обывателю, так и любой компании. С их помощью можно реализовать практически любой трудовой процесс, обмениваться рабочей информацией, реорганизовывать большинство видов деятельности, но данный вид взаимодействия, к сожалению, никогда не заменит живого общения.

Также недостатком электронного взаимодействия является ограниченное информационное пространство, которое влечет за собой неполную картину восприятия субъектов, их речи, интонации, позы, движений и т.д. в комплексе. В такой ситуации возникает множество упущений и недопониманий, которые отсутствовали бы или были бы исправлены при других обстоятельствах. Для того чтобы руководитель мог получать как можно больше детализированной информации, в электронной среде ему необходимо прикладывать значительно больше усилий и тратить дополнительное время для достижения аналогичных результатов.

Необходимо вспомнить, что система управления персоналом - это достаточно сложный, многоуровневый процесс, включающий в себя огромный спектр разнонаправленной деятельности. Переход на дистанционное управление персоналом производит необходимость перевода в дистанционный режим всех составляющих элементов упомянутой системы. Таким образом, необходим дистанционный поиск и отбор персонала, дистанционная адаптация персонала, дистанционное обучение, дистанционная оценка персонала и др. Дистанционно придется стимулировать персонал, дистанционно планировать его карьеру и, соответственно, формировать дистанционную корпоративную культуру.

В интернет-пространстве на сегодняшний день существуют огромные возможности для взаимодействия с потенциальными работниками. Множество электронных порталов на безвозмездной и материальной основе создают возможность для формирования и коллекционирования резюме. Любой работодатель вправе использовать данную информационную среду и формировать контакты по своему желанию и возможности. С другой стороны, возможно не менее эффективное размещение рекламной информации о потребности в сотрудниках на ту или иную должность. Собственный портал организации или специальные информационные среды, предназначенные для размещения такого рода данных, - выбор работодателя.

Вторым этапом может быть обмен документами с последующим их изучением. Данный процесс осуществляем по электронной почте, в различных приложениях, имеющих функции передачи и просмотра файлов. Te же WhattsApp и Telegram легко синхронизируются с ПК и другими подобными устройствами. Необходим анализ представленных источников информации, данных анкет, тестов и собственно самого резюме.

Третий этап уже достаточно давно используется крупными современными компаниями как промежуточный, а в сложившейся ситуации имеет права позиционироваться как основной - это ZOOM-собеседование, проведение видео-интервью с отделом кадров и непосредственным руководителем. После всех этих мероприятий обычно принимается решение о приеме на работу.

В цифровой среде существуют возможности для создания электронного документооборота. Программное обеспечение позволяет работать с документом одновременно нескольким сотрудникам и видеть, кто конкретно в данный момент им занимается. С помощью программных функций настраивается электронная передача документов, а также электронные процедуры подписания и нанесения оттиска печати. Для их реализации необходимо наличие электронно-цифровых подписей и печатей у сотрудников организации. Недостатком данного процесса является стоимость составных элементов и их временная ограниченность.

Дистанционная адаптация также имеет ряд особенностей. В первую очередь возникает острая необходимость в формировании внутренних коммуникаций, что затрудняется отсутствием офиса, а, следовательно, ответов на множество очень важных вопросов. Адаптация дистанционно сопровождается проведением онлайн опросов и интервью, знакомством 
с организацией посредством сайта, фильма и др. Нового сотрудника необходимо дистанционно познакомить с правилами, распорядком, организационной структурой, руководством и коллегами, обозначить необходимые контакты. Эффективным будет проведение приветственного тренинга в режиме онлайн либо вебинара с видеозаписью. Эффективным элементом было бы наличие закрытой корпоративной платформы для сотрудников, внутри которой реализовывалась бы возможность обмена информацией, документами, а также работа в группе и команде.

Для организации дистанционное обучение имеет ряд положительных особенностей:

- технологичность. С помощью инструментов онлайн связи (электронная почта, Skyp, Zoom, облачные хранилища) преподаватели могут своевременно обеспечивать обучающихся учебными материалами и заданиями, а обучающиеся, в свою очередь, - предоставлять ответы в письменной и устной форме;

- доступность и открытость. Обучение в любое удобное неограниченное время и без отрыва от производства;

- стоимость. По сравнению с другими формами образования дистанционный формат наиболее экономный [4].

Рассмотрим опыт использования дистанционных инструментов оценки.

1. Выбор компетенций. Дистанционный формат дает хорошие возможности для оценки компетенций, в основе которых лежат аналитические навыки (собственно анализ, стратегическое видение, планирование и организация, работа с изменениями и т. п.), позволяет получать дополнительную информацию для оценки коммуникативных навыков и отчасти уровня мотивации, нацеленности на результат.

2. Выбор упражнения. Проблемы, заданные в упражнении, должны соотноситься с текущими задачами участников, но не повторять их буквально, быть сложными, но при этом выполнимыми. Правильный кейс выявит именно те особенности поведения/подхода, которые помогают или мешают сотрудникам быть эффективными.

3. Описание проявления поведения. Один из ключевых моментов - формализация требований к поведению, соответствующему той или иной оценке («растяжка по шкале»). Наверное, это наиболее трудоемкий и кропотливый этап, но именно в нем залог успеха всего проекта, ведь это существенно упрощает анализ собранной информации, задает единые критерии оценки и в дальнейшем позволяет дать обоснованную обратную связь участникам.

4. Подготовка экспертов. Как правило, в масштабных проектах по оценке в роли эксперта приходится выступать людям с очень разным уровнем подготовки, с опытом оценки или без него, но команде асессоров крайне важно придерживаться общих стандартов и технологии. Отсюда три обязательных шага подготовки: бриф по упражнению для всей команды, тренинг наблюдателей для новичков, интеграционная калибровочная сессия для отработки навыков оценки и согласования критериев на примере реального кейса $[6,7,8]$.

Заключение. Система управления персоналом в условиях дистанционной формы реализации ее основных функций имеет множество специфических особенностей. Современные организации практикуют дифференцированный подход в управлении «удаленным персоналом», реализуя огромный спектр разнонаправленной деятельности. Важной проблемой, которая может существенно повлиять на качество дистанционного управления, является прямая зависимость от наличия и исправности технических средств, постоянной подачи электроэнергии и бесперебойной работы сети Интернет. Эти факторы являются объективными и порой создают ситуации, когда субъект не имеет физической возможности выполнить работу и предоставить ответы вовремя, что также негативно сказывается на психологическом состоянии сотрудников во время обучения и может вызывать сильные беспокойства, неврозы и истерики.

\section{Литература}

1. Gafiatulina N. Kh., RachipaA.V., Vorobyev G.A. et al. Socio-political changes as a socio-cultural trauma for the social health of Russian youth // Modern Journal of Language Teaching Methods. - 2018. - Vol. 8. №5. - Pp. 602-609.

2. Бурмистров С. В., Волочай А. В., Самыгин С.И. Мотивы трудовой деятельности в системе управления персоналом организации // Гуманитарные, социально-экономи- 
ческие и общественные науки. - 2019. №9. - С. 26-29.

3. Рачипа А.В., Бурмистров С.В., Самьгин С.И. Ценностно-смысловые ориентации руководителя как фактор повышения качества управления образовательной организацией // Государственное и муниципальное управление. Ученые записки. - 2020. №1. - C.251-255.

4. Руденко А.Н. Интеллектуальный потенциал студенчества региональных вузов. В сборнике: Модернизация экономики России: отраслевой и региональный аспект материалы международной научно-практической конференции профессорско-преподавательского состава, молодых ученых и студентов. - 2018. - С. 352-356.

5. Сипатова M. Рецепты эффективности на каждый день: секреты дистанционного управления // Новая аптека. — 2012. — №9-1. C. 58-65.

6. Тарабардина Н. Дистанционная оценка персонала: возможности и проблемы. Опыт реализации проекта. // Управление персоналом. - 2018. - №41.

7. Popov A. V., Rachipa A. V., Abazieva K. G., Shvets L. G., Kobysheva L.I., Goncharova S.N., Samygin S.I. Specificity of explication of the category «management decisions» and scientific-methodological approaches for their development // Revista Inclusiones. - 2019. Vol. 6. - №2. - Pp. 215-224.

8. Руденко А.М., Котлярова В.В., Самысгин С.И., Попов А.В., Дорошенко А. Ю., Латылшева А.Т. Управление персоналом. Ростов-на-Дону, 2020.

\section{Referense}

1. Gafiatulina N. Kh., Rachipa A.V., Vorobyev G.A. et al. Socio-political changes as a socio-cultural trauma for the social health of Russian youth // Modern Journal of Language Teaching Methods. - 2018. - Vol. 8. №5. - Pp. 602-609.

2. Burmistrov S.V., VolochajA.V., Samygin S.I. Motivy trudovoj dejatel'nosti v sisteme upravlenija personalom organizacii [Motives of labor activity in the organization's person- nel management system] // Gumanitarnye, social'no-jekonomicheskie i obshhestvennye nauki [Humanities, socio-economic and social Sciences]. — 2019. — №9. — Pp. 26-29.

3. Rachipa A.V., Burmistrov S.V., Samygin S.I. Cennostno-smyslovye orientacii rukovoditelja kak faktor povyshenija kachestva upravlenija obrazovatel'noj organizaciej [Value-semantic orientations of the head as a factor of improving the quality of management of an educational organization] // Gosudarstvennoe i municipal'noe upravlenie. Uchenye zapiski [State and municipal management. Scientific notes]. - 2020. — №1. - Pp. 251-255.

4. Rudenko A.N. Intellektual'nyj potencial studenchestva regional'nyh vuzov. V sbornike: Modernizacija jekonomiki Rossii: otraslevoj i regional'nyj aspekt materialy mezhdunarodnoj nauchno-prakticheskoj konferencii professorsko-prepodavatel'skogo sostava, molodyh uchenyh i studentov [Intellectual potential of students of regional universities. In the collection: Modernization of the Russian economy: industry and regional aspect materials of the international scientific and practical conference of teaching staff, young scientists and students]. 2018. - Pp. 352-356.

5. Sipatova $M$. Recepty jeffektivnosti na kazhdyj den': sekrety distancionnogo upravlenija [Efficiency recipes for every day: secrets of remote control] // Novaja apteka [New pharmaсу]. — 2012. — №9-1. — Pp. 58-65.

6. Tarabardina N. Distancionnaja ocenka personala: vozmozhnosti i problemy. Opyt realizacii proekta [Remote staff assessment: opportunities and challenges. Experience in the implementation of the project] // Upravlenie personalom [Personnel management]. — 2018. - №41.

7. Popov A. V., Rachipa A. V., Abazieva K. G., Shvets L. G., Kobysheva L.I., Goncharova S.N., Samygin S.I. Specificity of explication of the category «management decisions» and scientific-methodological approaches for their development // Revista Inclusiones. - 2019. Vol. 6. - №2. - Pp. 215-224.

8. Rudenko A.M., Kotljarova V.V., Samygin S.I., Popov A.V., Doroshenko A. Ju., Latysheva A.T. Upravlenie personalom [Personnel management]. - Rostov-on-Don, 2020. 


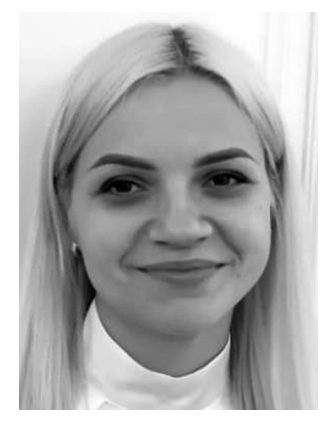

Руденко Анна Николаевна - кандидат социологических наук, доцент кафедры «Управление персоналом и социология» Ростовского государственного экономического университета (РИНХ).

Rudenko Anna Nikolaevna - Candidate of Sociological Sciences, Associate Professor, Department of Personnel Management and Sociology, Rostov State University of Economics.

344082, г. Ростов-на-Дону, пер. Островского, 62, каб. 204 62 per. Ostrovsky, off. 204, 344082, Rostov-on-Don, Russia E-mail: anya_rudenko@bk.ru 